\title{
Simulation of Universal Design by a Functional Design Method and by Gamification of Building Information Modeling
}

\author{
Jukka Selin \\ Department of Electrical Engineering and Information \\ Technology, Mikkeli University of Applied Sciences \\ Ltd Patteristonkatu 2, 50100 Mikkeli FINLAND \\ jukka.selin@mamk.fi
}

\author{
Markku Rossi \\ Department of Electrical Engineering and Information \\ Technology, Mikkeli University of Applied Sciences \\ Ltd Patteristonkatu 2, 50100 Mikkeli FINLAND \\ markku.rossi@mamk.fi
}

\begin{abstract}
We have developed a method and a process with which we can simulate the functionality and the space requirements of different actions in buildings. We utilize Gamification of Building Information Modeling (BIM). The essential goal of Building Design is to produce designs corresponding to customers' needs. In our method the electronic CAD model is inserted into a Game Engine and is gamified. Features and limitations that correspond to the reality as exactly as possible are generated for the virtual objects steered in the game by the designers. In addition, the space requirements for the actions performed by a simulated user can be dimensioned by our Functional Design Method. We generate 3D space objects of maximum space requirements for actions from videos of real human actions. The $3 D$ space objects created this way can be Collider Objects used by the designers attending to the Gamification. We get simulated User Experiences from the rooms under design. This method helps to understand better than before different end users and their needs. The result is buildings that fit the requirements of the users.
\end{abstract}

\section{INTRODUCTION}

$\mathrm{T}$ he Pilot Experiments described in this article use our method to generate computer game like environments out of a Building Information Models (BIM) by Gamification. The gamified Building Model enables us to simulate actions in buildings.

With the aid of the gamified model we can simulate different User Experiences in a building already when the building is under design.

The "Players" following different roles can move like the First Person Controller (FPC) or the Third Person Controller (TPC) in the virtual space. Features and limitations according to a role (e.g. a Handicapped using a wheelchair) can be realised.

The Game Object can include the space requirements belonging to the actions of the role. The space requirements are from our Functional Design Method, FDM [1],[2]. From the space requirements we can generate variable Collider Functions around the Game Object. The method makes it possible to simulate different functionalities and space requirements related to actions. The Player can e.g. take the role of a person using a rollator. With the aid of the gamified
Building Model he can get the User Experience of moving around with a rollator. We detect already in the beginning the possible problems and shortcomings, and corrections are then low coast and fast compared to during the construction or even after the building project.

The developed method also helps designers of the Construction Industry to better understand the needs and limitations of buildings. It is also possible to involve the user groups to the design via the method of gamified Building Model. The end users can get a view that is understandable and real enough. We can then achieve buildings that match more closely their purpose.

With the method it is also possible to automate the testing against the Universal Design principles, by moving 3Dmodels with their space requirements (like a wheelchair patient with two assistants) automatically through the model. After the tests the possible problems and their locations can be found in the report automatically generated.

If the designer uses VR glasses, like Oculus Rift, the User Experience of the gamification of Building Information Models can be quite realistic.

The gamification of the models serves all phases of the construction project from sales and marketing until the completion of the Architectural Design. By the aid of the gamified model it is possible to communitise and crowdsource by distributing the gamified model in the Internet. The end users can then be given an opportunity to present ideas, test functionalities and so attend to the design.

This article first describes the methodologies and software related to the research. Then we present the new method and the process for gamification of Building Information Models as an aid in design. Finally, we observe the meaning of the new results and scenarios for the next steps.

\section{Methodologies And SOFtware USEd IN The Research}

The developed method utilises a work based invention report at Mikkeli University of Applied Sciences Ltd (Mamk) filed by Jukka-Pekka Selin. The maximum space requirement in dimensions $\mathrm{x}, \mathrm{y}$ and $\mathrm{z}$ can be derived from video clips of real human actions. In this method the real actions are videoed with at least two video cameras that are situated at right angles. The need of space from the actions in three 
dimensions is measured and a corresponding geometrical object is created. This $3 \mathrm{D}$ object in IFC file format is compatible with CAD software. The goal is to ensure that the required actions can fit the space under design. [1],[2],[3].

During the research program the application that is BIM compatible, under development and is meant for Lifecycle Management of Building Data, and the application extension Value Add Data of Mamk R\&D, were used for creating 3D objects. The application extension realizes the functionality of the FDM. The Colliders describing the space requirements for different actions to the gamified model were created via the execution of the Value Add Data software.

The 3D-model that contains a Building Model was realised with the ArchiCAD design software and it was gamified with the Unity Game Engine.

\section{The FDM together with the GAmificAtion OF the \\ BUILDING INFORMATION MODELS ENHANCE THE QUALITY, VISIBILITY AND UNDERSTANDABILITY IN DESIGN}

When the practices of BIM develop, new ways to design, visualise and analyse buildings are developed in parallel. The earlier the issues are detected and corrected, the lower are the costs and related work efforts. The FDM we developed earlier is a method and process born out of need. It can be applied very well in parallel with BIM, and could even be standardised to be an integral part of it.

The FDM can be the basis for developing new analysis functions, techniques and methods to support the design process and the designers.

We combined the FDM to the gamification of the Building Models and did research on whether it could be possible to create User Experiences about how the usage of e.g. a wheelchair or a rollator succeeds in the spaces under design.

With the aid of the 3D space objects from the FDM we can generate e.g. Colliders around the functional unit (e.g. a wheelchair user with two assistants). Collider is a term used in Game Design. The Collider then corresponds to the measured space requirements of the human action. In the First Person Controller concept the Colliders created this way can be attached to the virtual model of a wheelchair Use Case. After this we could move around with the wheelchair in the gamified Building Model and swap the Collider according to the actions the handicapped and his assistants were doing.

When VR glasses like Oculus Rift are used, we got a very realistic User Experience about how it would feel to move and perform different kinds of functions with a wheelchair in a real building.

Our partners agreed this kind of visualisations and generation of User Experiences are one of the directions for forcefully developing BIM and its applications. This kind of approach is quite new in the relatively conservative Contruction Industry, but we think it will become more common.
The virtualisation of buildings and gamification open new perspectives to the whole construction business and the design processes used there. It e.g. enables to involve the future users to the idea gathering phase and to the design work in ways not possible earlier.

\section{A. The space required by Human Actions is converted into a $3 D$ object by the FDM}

The design needs described earlier get means of enhancement from the FDM from Phil.Lic. Jukka-Pekka Selin. The method helps especially in a situation where the building operations are not yet active but there exists a digital model of the building.

In addition to a mere idea we offer a ready process that enables to transfer the space requirements of real Human Actions to the BIM based designs via our 3D IFC-objects. It is possible to test and simulate with the Building Model how the functional demands fit the rooms under development. The diagram below shows the process of the FDM:

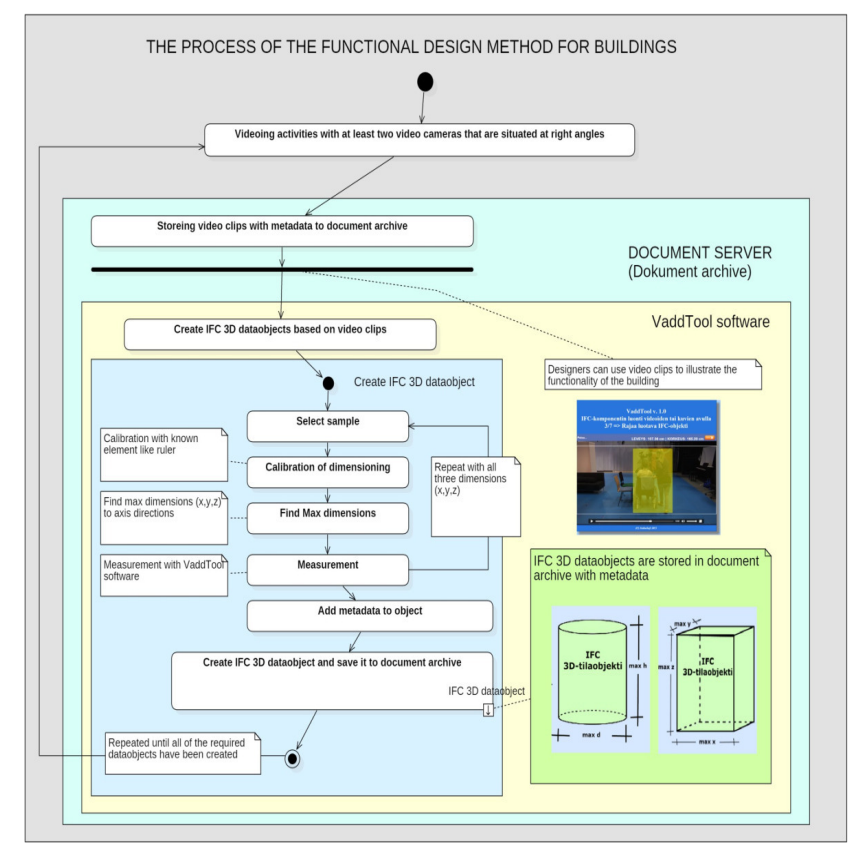

Fig 1. The process of the FDM for Buildings.

An example of the meant human activities is "an assistant who helps a wheelchair user to dress on outdoors clothes". The invention to use real human activities and turn them into 3D CAD objects is from Licentiate of Philosophy JukkaPekka Selin who is the principal lecturer of data processing at Mamk.

Fig. 2 shows how the dimensioning $(x, y, z)$ of the forthcoming IFC data object is performed. 


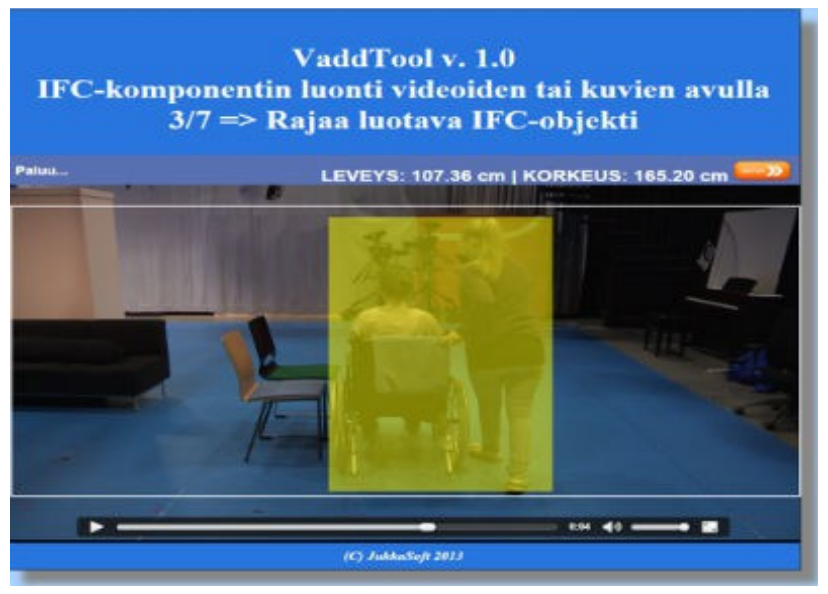

Fig 2. A screen shot of the Space Required by Activity dimensioning function of the Value Add Data software prototype.

\section{B. The Gamification of Building Models with the Unity Game Engine}

We realised a pilot project at Mamk where we gamified the building model of the headquarters of the construction company U.Lipsanen Oy. The foundation was the 3D-model from ArchiCAD software. It was a part of the building model [4]. The Unity Game Engine supports importing data in various 3D file formats. After iterative testing and piloting we ended up to recommend that the import format should be the FBX format developed by Autodesk, Inc. [5]. Unity has native FBX support.

\section{Simulating the actions of a handicapped building user}

We piloted the methods by simulating a wheelchaired, moving person. The space requirements for different actions where first generated by the FDM. The original material was a set of video clips about real actions that were input to our Value Add Data software for creating IFC files.

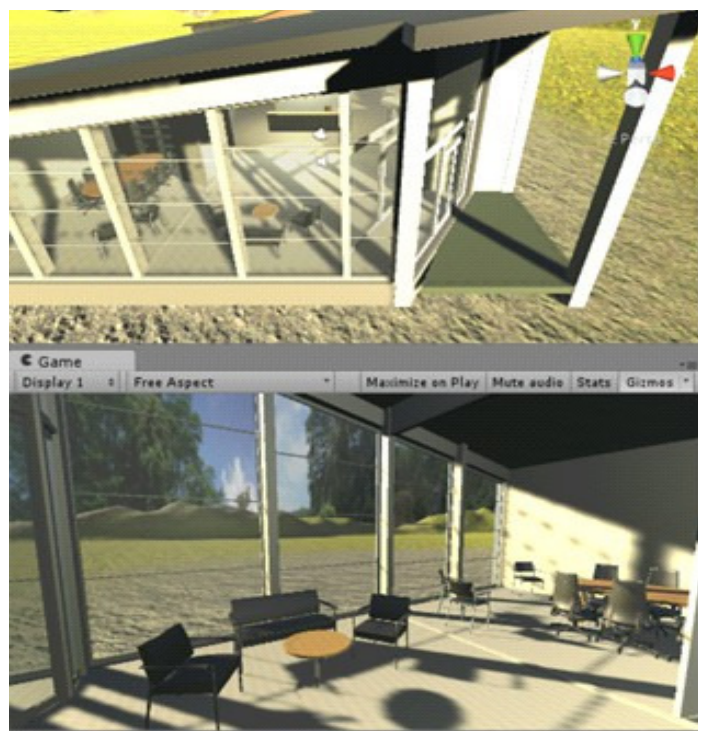

Fig 3. A 3D building model converted first to FBX has been imported to the Unity Game Engine.
In the pilot we created a Third Person Controller-type Player who moved with a wheelchair. As much realism as possible was programmed for the dimensions, the movements and the building automation. We also created swappable Colliders around the virtual player. This way the space requirements can be swiftly selected according to the action under test. We can see and experience in a concrete way how well the action fits the spaces under design.

The dimension data from the FDM, representing the space need of a real action, can be utilised as such when creating Colliders in the Game Engine. One of the future research topics is to develop an automated creation procedure of Colliders based on the 3D IFC files. The next table below shows as an example the space requirements of two actions studied in piloting.

TABLE I.

MAXIMUM SPACE REQUIREMENTS FOR THE DIMENSIONING OF THE COLLIDERS CREATED BY THE FDM

\begin{tabular}{|l|c|}
\hline The dimensioned Action (VaddTool) & Space Requirement (m) \\
\hline $\begin{array}{l}\text { The wheelchair user is helped to dress } \\
\text { on or off his shoes by his assistant }\end{array}$ & $1.10 \times 1.90 \times 1.51 \quad(\mathrm{x}, \mathrm{y}, \mathrm{z})$ \\
\hline $\begin{array}{l}\text { The assistant walks with the } \\
\text { wheelchair user on the side or behind } \\
\text { the wheelchair }\end{array}$ & $1.07 \times 1.90 \times 1.30(\mathrm{x}, \mathrm{y}, \mathrm{z})$ \\
\hline
\end{tabular}

Respectively it is possible to dimension and simulate all kinds of actions, also not related to Building Design, by using the Functional Design Method and Gamification of 3D Models. There is a lot of potential in generalisation.

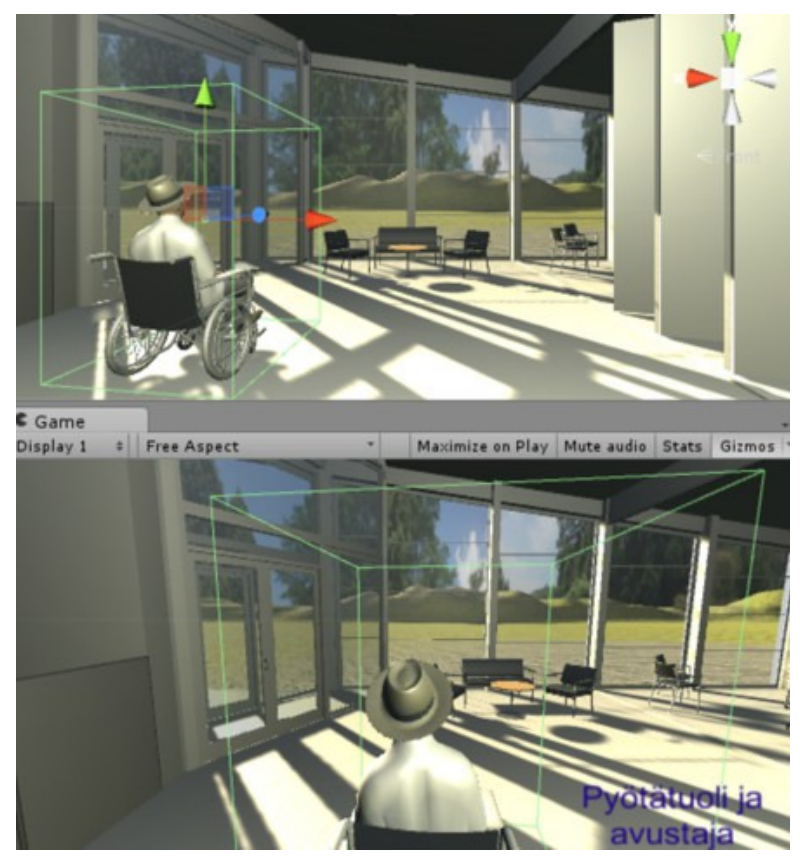

Fig 4. A handicapped virtual player with an added Collider Box (in green) derived with the FDM. Respectively it is possible to dimension and simulate all kinds of actions, also not related to Building Design, by using the FDM and Gamification of 3D Models. 


\section{Work process oriented, location aware document} management and viewing services for the whole Lifecycle

After testing the creation of 3D IFC objects and short term document storage with the Value Add Data software prototype we began to negotiate with the public financiers about developing a service that would manage the documents during the whole lifecycle of buildings. In December 2015 the construction company U.Lipsanen Oy won a partial public financing for their development project RedHal.

The new targeted functionality differs from the presently available services in several features. After the documents reach the status "As built - Ready ", we will move the documents to a long term data repository with a targeted service life of 100 years. The University is an expert in Data Migration through storage technologies and in semantic digital archiving [7].

We are targeting to use indoor positioning in the 0.5 metre accuracy range together with tablets and Augmented Reality services. The data structures both in the Pre As built service and in the long term repository are according to the semantic structures of buildingSMART.

The document viewing will be of an active type. It takes into account the role of the viewer, the location, the orientation and the current phase of the design or construction process. The co-operation between designers will be based on Building Models according to the BIM standards. This project will be finalised by June 2018 .

\section{Results AND Conclusions}

The following picture describes the process aspect in piloting our method.

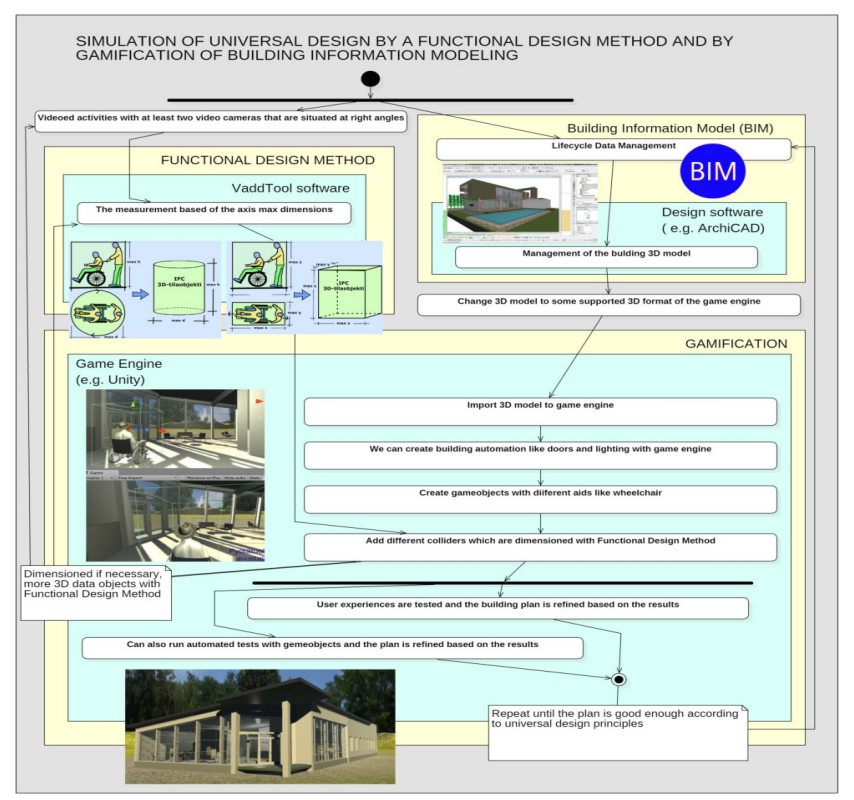

Fig 5. The process developed at Mamk in simulation of Universal Design with the aid of a gamified Building Model.
The results from the piloting are promising. The User Experience in the gamified model can be highly natural. The attendants of the Design Game thought that by bringing a handicapped virtual player to the game, a demonstrative and mind-expanding understanding was created about how well the building corresponds to users' needs.

Their opinion was that the method helps any designer to take the position of a handicapped user and this way to understand their needs and take them into account. The method also helps to test how well the building automation (e.g. active doors and adaptive lighting) serve different users. The FDM was found during the piloting also to be practical in ensuring that the spaces are dimensioned according to real needs. The idea behind the developed method about FDM based Colliders for the Players in a simulation based on gamified Models was found to be good and practical.

We also studied the automating of dimensioning testing by moving Players with Space Requirement Colliders automatically through pre-selected rooms in a way that the collisions and reroutings were documented to a log. Now we saw at once the locations which were too small or narrow for the required actions. It was also possible to study the steepness of ramps. The partners said the gamification of Building Models and the virtualisation of the usage of buildings create new and necessary dimensions to the whole building design process and we have the right future direction in our research. The development of Design Processes can now move towards crowdsourcing and communitisation by bringing the gamified Building Model e.g. via the WebGL technology to the Net and by enabling the future users of buildings access to the preliminary designs, for commenting. We think these processes can help in creating buildings that really fit the users' needs.

\section{REFERENCES}

[1] Toimintatilan määrittävä objekti tietokoneavusteista suunnittelua varten. Finnish patent application 20135286FI. Applicant Mikkeli University of Applied Sciences Ltd (Jukka-Pekka Selin).

[2] An action space defining object for Computer Aided Design. PCT patent application WO 2014/154942 A1. Applicant Mikkeli University of Applied Sciences Ltd (Jukka-Pekka Selin).

[3] Rossi, Markku J., Dave, Bhargav., Digitalization and quality enhancement initiatives in sw assisted design processes in building and construction industries. 9th International Conference on Computer Engineering and Applications, Dubai, 2015. ISBN 978-161804-276-7.

[4] Rakennusliike U.Lipsanen, Rakennusliike U.Lipsanen Oy Headquarters building (Lipatie 1, 76850 Naarajärvi). A 3D Model designed with ArchiCAD saved in the 3DS format. Contruction Company U.Lipsanen Oy, Pieksämäki, Finland, 2015.

[5] Autodesk Inc. The official website of the Autodesk FBX format. Accessed on March 30, 2016. http://www.autodesk.com/products/fbx/overview

[6] Autodesk Inc. The official website of the FBX Converter software. Accessed on March 30, 2016. http://usa.autodesk.com.

[7] Uosukainen, Liisa (ed.), Open Source Archive. Towards open and sustainable digital archives. Mikkeli University of Applied Sciences, Series A, No. 94, 2014. 100 pp. http://urn.fi/URN:ISBN:978-951-588456-5. Accessed on May 9, 2016. 